\title{
CCD Inhibition Test Can Improve the Accuracy of the Detection of Pollen and Seed Food Allergen-Specific IgE in Southern China
}

\section{Wenting Luo* \\ Huimin Huang* \\ Peiyan Zheng \\ Jinping Zheng (D) \\ Baoqing Sun (D)}

Department of Allergy and Clinical Immunology, State Key Laboratory of Respiratory Disease, National Clinical Research Center for Respiratory Disease, Guangzhou Institute of Respiratory Health, First Affiliated Hospital of Guangzhou Medical University, Guangzhou Medical University, Guangzhou, People's Republic of China

*These authors contributed equally to this work
Correspondence: Baoqing Sun; Jinping Zheng

Department of Allergy and Clinical Immunology, State Key Laboratory of Respiratory Disease, National Clinical Research Center for Respiratory Disease, Guangzhou Institute of Respiratory Health, First Affiliated Hospital of Guangzhou Medical University, Guangzhou Medical University, I5 I Yanjiang Road, Guangzhou, Guangdong Province, People's Republic of China Tel +86-20-8306-2865

Email sunbaoqing@vip.163.com; 18928868238@|63.com
Objective: The presence of cross-reactive carbohydrate determinants (CCDs) may cause false-positive results in vitro allergen sIgE tests. In this paper, we focused on pollen sensitisation and its relationship with CCD in patients with respiratory allergic diseases in South China. A CCD inhibition test was conducted to assess whether patients were truly allergic to pollen or whether their sIgE was caused by a CCD cross-reaction, thus providing an important basis for clinical diagnosis and treatment.

Methods: Patients with known serologic pollen sensitization were selected, and sIgE of mugwort, tree mix 20 (willow/poplar/elm tree), common ragweed, Humulus scandens, peanut, soybean and CCD was detected via the EUROBlotMaster system. Thirteen CCD-sIgE negative patients and 33 CCD-positive patients were selected, and their serum samples were subjected to the CCD inhibition test.

Results: We found that $66.0 \%$ to $95.9 \%$ of patients sensitised to pollen and seed food allergens were co-sensitized to CCD. Additionally, $73.0 \%$ to $100 \%$ of the sIgE tests for pollen and seed food allergens turned negative after inhibition, mostly for allergens from Humulus scandens $(100 \%, 15 / 15)$, followed by mugwort and peanut $(85.2 \%, 23$ / $27)$, ragweed $(81.5 \%, 22 / 27)$, soybean $(80.0 \%, 20 / 25)$, and tree pollen $(73.0 \%, 19 / 26)$. Conclusion: CCD causes false positives in the in vitro allergen $\operatorname{sIgE}$ tests of patients with respiratory allergic diseases in South China. Attention should be paid to the use of CCD inhibitors in diagnosing in vitro allergies because of their importance in diagnosing and treating local allergic diseases.

Keywords: pollen allergen, food allergen, false-positive, cross-reactive carbohydrate determinants, CCD, special IgE, EUROBlotMaster

\section{Introduction}

Allergen sensitisation is usually identified by the allergen-specific $\operatorname{IgE}$ (sIgE) test and skin allergy test (eg, intradermal and skin prick test). The serum allergen sIgE test based on fluorescein immunoassay, chemiluminescence immunoassay and enzyme immunoassay is often favoured in clinical application because of its ability to detect $\operatorname{sIgE}$ for different allergens and its high specificity. ${ }^{1,2}$ However, in some cases, despite finding positive results for the allergen $\operatorname{sIgE}$, patients often show no clinical symptoms. Some scholars have recently pointed out that this phenomenon may be due to clinically irrelevant $\mathrm{IgE}$ antibodies against $\mathrm{CCD} .{ }^{3,4}$ Clinicians may prescribe inappropriate dietary restrictions or avoidance recommendations, as well as excessive drugs or 
immunotherapy, based on false-positive test results for the allergen $\operatorname{sIgE} .^{5}$ The presence of CCD-sIgE can be detected by specific CCD allergen tests in vitro. A positive CCD-sIgE result, on the other hand, only confirms the presence of CCD-sIgE antibody in the serum and is not a reliable criterion for determining whether the response to a specific allergen is caused solely by CCDs or also by protein epitopes. The removal of CCD-sIgE by solidified CCD antigen has also been proposed, but this procedure is too complex to be applied in clinical testing. ${ }^{6,7}$ According to the guidelines for in vitro allergy diagnosis developed by the Association of Scientific Medical Societies in Germany (AWMF), allergen inhibition tests using CCD inhibitors can significantly improve the specificity of sIgE detection. ${ }^{8-10}$

In our previous study, we found that the positivity rate for pollen $\operatorname{sgE}$ among allergic rhinitis patients in southern China was approximately $20 \%$. The majority of these patients had serum pollen sIgE concentrations lower than $17.5 \mathrm{kUa} / \mathrm{L},{ }^{11}$ and they frequently did not have seasonal allergy symptoms. In this study, we further studied pollen allergens and their relationships with CCD in patients with respiratory allergic diseases in South China. To provide appropriate guidance for clinical diagnosis and treatment, we used the CCD inhibition test to determine whether patients in southern China were truly allergic to pollen or whether the positive sIgE result was caused by CCD cross-reaction.

\section{Materials and Methods}

\section{Study Population}

170 outpatients with symptoms of allergic rhinitis from the Allergy Center of the First Affiliated Hospital of Guangzhou Medical University were screened between January 2017 and December 2018. The included patients fulfilled the following criteria: (1) a physician diagnosis of asthma, ${ }^{12}$ rhinitis, ${ }^{13}$ or chronic cough; and (2) positive sIgE reactivity to two or more indoor and outdoor inhalant allergen extracts, such as house dust mite, cockroach, mould mix, Humulus scandens, dog dander, wheat, ragweed, mugwort, and tree mix, as recorded by previous ImmunoCAP results. The exclusion criteria were immunodeficiency and a history of treatment with allergen-specific immunotherapy. The average age of the 170 patients with multi-allergen sensitisation was 26 (range 1-84) years; 41.8\% (71/ 170) were children ( $\leq 14$ years old, $(6,1-13$ years)), and $58.2 \%(99 / 170)$ were adult $(>14$ years old, (43, 16-84)).

The study was carried out with the approval of the ethics committee of the First Affiliated Hospital of Guangzhou Medical University (GYFYY-2017-18). This study was conducted in accordance with the Declaration of Helsinki. Written informed consent was collected from all the patients. For subjects younger than 18 years of age, written informed consents were obtained from their parents or legal guardian.

\section{Determination of Specific IgE Antibodies}

SIgE antibodies to mugwort, tree mix pollen (willow/ poplar/elm tree), common ragweed, Humulus scandens, peanut, soybean and CCD were detected using the EUROBlotMaster system (EUROIMMUN Medizinische Labordiagnostika AG, Lübeck, SH, Germany) according to the manufacturer's instructions. The cut-off value for positive test results was 0.35 $\mathrm{kUa} / \mathrm{L}$. The $\mathrm{sIgE}$ reactivity was categorized quantitatively into six classes: Class $1(\geq 0.35$ to $<0.70 \mathrm{kUa} / \mathrm{L})$, Class 2 ( $\geq 0.70$ to $<3.50 \mathrm{kUa} / \mathrm{L}$ ), Class 3 ( $\geq 3.50$ to $<17.50 \mathrm{kUa} / \mathrm{L})$, Class $4(\geq 17.50$ to $<50.00 \mathrm{kUa} / \mathrm{L})$, Class 5 ( $\geq 50.00$ to $<100.00 \mathrm{kUa} / \mathrm{L})$, and Class 6 $(\geq 100.00 \mathrm{kUa} / \mathrm{L}){ }^{11,14,15}$ Patients with plant allergen sensitisation (mugwort, tree mix, common ragweed, Humulus scandens, soybean and peanut; sIgE positivity for one of these allergens was defined as plant allergen sensitisation (PAS)) were evaluated to determine the correlations between pollen allergens and plant food allergens and analyse the effect of CCD on plant allergen sensitisation.

\section{Cross-Inhibition Assay}

We selected PAS patients from the above cases, including $33 \mathrm{CCD}$-sIgE positive and $13 \mathrm{CCD}$-sIgE negative patients. Their sera were re-incubated in the EUROLINE assay using the anti-CCD Absorbent (product classification DQ, DP). The CCD inhibitor was composed of glycopeptides derived from pineapple stem bromelain.

CCD blockers and sera were diluted with the same buffer. For each sample to be tested, the sample 
volume $(300 \mu \mathrm{L})$ was mixed with the corresponding amount of anti-CCD Absorbent $(15 \mu \mathrm{L})$ according to the manufacturer's instructions, resulting in a final concentration of $20 \mu \mathrm{g} / \mathrm{mL}$. Anti-CCD Absorbent was pipetted into the sample, and the preparation was shaken for 60 minutes at room temperature $\left(+18^{\circ} \mathrm{C}\right.$ to $+25^{\circ} \mathrm{C}$ ). Following that, the sera were processed using EUROLINE for allergens and CCD.

\section{Statistical Analysis}

SPSS 22.0 was used to analyse the data (SPSS, Chicago, IL). Categorical variables are shown as numbers and percentages of the total. Categorical variables were analysed by the chi-square test $\left(\chi^{2}\right)$ or Fisher's exact probability method. Radar charts were used to compare different characteristics of plants allergen SIgE in CCD-sIgE negative or CCD-sIgE positive patients. The correlations between allergens and CCD were calculated with optimal scale analysis. A value of $P<0.05$ was considered statistically significant.

\section{Results}

\section{Distribution of slgE Against Plant} Allergens and CCD in 170 Polysensitised Allergic Rhinitis Patients

Among the 170 individuals, 112 (65.9\%) were sIgEpositive for at least two pollen or plant food allergens, and $79(46.5 \%)$ were positive to CCD-sIgE. The positivity rates and levels of sIgE to pollen and food allergens detected by EUROBlotMaster system, are shown in Table 1 and Figure 1. The sIgE positivity rates from high to low were soybean $(55.3 \%, 94 / 170)$, tree pollen $(52.4 \%, 89 / 170)$, peanut $(51.2 \%, 87 / 170)$, mugwort $(48.8 \%, 83 / 170)$, common ragweed $(42.9 \%, 73 / 170)$, and Humulus scandens $(30.0 \%, 51 / 170)$ (Table 1). The proportions of adult patients sensitised to PAS $(80.8 \%, 80 / 99$ vs $77.5 \%, 55 / 71)$ and CCD (48.5\%, 48/ 99 vs $43.7 \%, 21 / 71)$ were higher than that of children, but there were no statistically significant differences (both, $P>0.05$ ).

\section{Prevalence and Levels of slgE Against CCD and Plant Allergens}

Plant allergen sIgE levels in PAS patients were mostly class 1-3 (Figure 1), with concentrations less than 17.5
Table I Distribution of Plant Allergens and CCD in 170 Polysensitised Allergic Rhinitis Patients

\begin{tabular}{|c|c|}
\hline & $\begin{array}{c}\text { Polysensitised Allergic Rhinitis } \\
\text { Patients ( } \mathrm{N}=170)\end{array}$ \\
\hline $\begin{array}{l}\text { Age (years), median } \\
\text { (QI, Q3) }\end{array}$ & $26(6,45)$ \\
\hline $\begin{array}{l}\text { Age group, Children/ } \\
\text { Adult, } n\end{array}$ & $71 / 99$ \\
\hline Sex, M/F, n & $96 / 74$ \\
\hline $\begin{array}{l}\text { Diseases, n, (\%) } \\
\text { Asthma } \\
\text { Rhinitis } \\
\text { Chronic cough } \\
\text { Other }\end{array}$ & $\begin{array}{l}42(24.7 \%) \\
57(33.5 \%) \\
37(31.8 \%) \\
34(20.0 \%)\end{array}$ \\
\hline $\begin{array}{l}\text { Positive number, } \mathbf{n}(\%) \\
\text { Negative to plant } \\
\text { allergens } \\
\text { Single plant allergen } \\
\text { positive } \\
\text { Multiple plant allergens } \\
\text { positive }\end{array}$ & $\begin{array}{l}35(20.6 \%) \\
23(13.5 \%) \\
\text { II } 12(65.9 \%)\end{array}$ \\
\hline $\begin{array}{l}\text { Allergen positivity, n (\%) } \\
\text { CCD } \\
\text { Soybean } \\
\text { Peanut } \\
\text { Humulus scandens } \\
\text { Mugwort } \\
\text { Common ragweed } \\
\text { Tree mix pollen }\end{array}$ & $\begin{array}{l}79(46.5 \%) \\
94(55.3 \%) \\
87(51.2 \%) \\
51(30.0 \%) \\
83(48.8 \%) \\
73(42.9 \%) \\
89(52.4 \%)\end{array}$ \\
\hline
\end{tabular}

Notes: Children ( $\leq 14$ years), adult (>14 years).

Abbreviations: $M$, male, $F$, female.

$\mathrm{kUa} / \mathrm{L}$. We found that $95.9 \%(70 / 73)$ of patients sensitised to common ragweed were $\mathrm{CCD}$-sIgE positive, as were $79.8 \%(71 / 89)$ of patients sensitised to tree pollen, $76.5 \%$ (39/51) of patients sensitised to Humulus scandens, $73.6 \%$ $(64 / 87)$ of patients sensitised to peanut, $68.7 \%(57 / 83)$ of patients sensitised to mugwort and $66.0 \%$ (62/94) of patients sensitised to soybean.

\section{Effect of the Presence of CCD-slgE on Plant Allergens}

The positivity rates for plant allergens (tree mix, common ragweed, soybean, peanut, mugwort and Humulus 


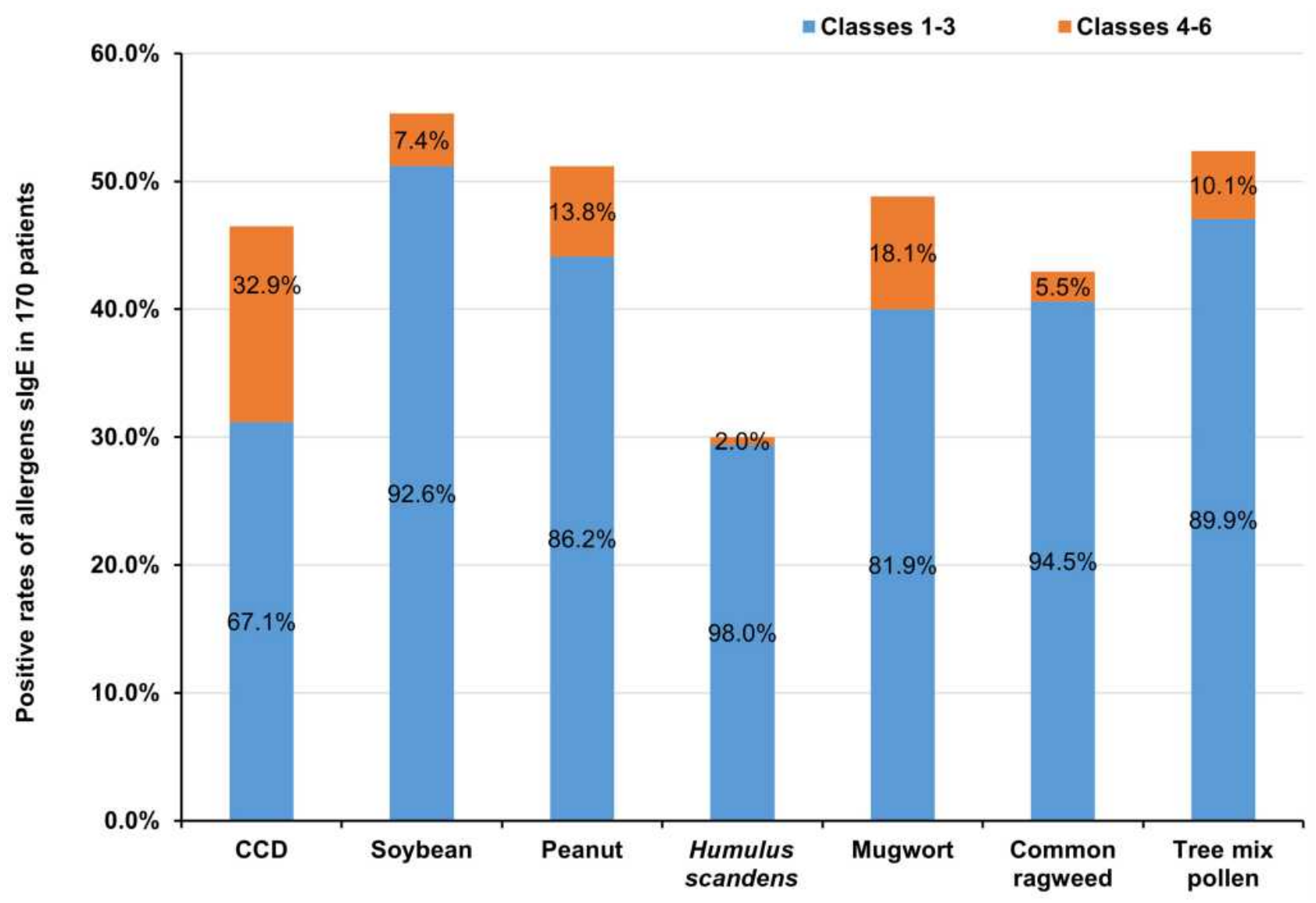

Figure I Prevalence rates and sIgE levels of CCD and plant allergens in 170 patients.

scandens) were significantly higher in CCD-sIgE positive patients than in CCD-sIgE negative patients (all $P<0.01$ ) (Figure 2A).

After stratifying patients based on their sIgE response to CCD (classes 1-3 and classes 4-6), they were evaluated for co-sensitisation to other pollen allergens and food allergens. SIgE reactivity to plant allergens was strongly associated with the presence of CCD-sIgE. Among the patients with classes 1-3 CCD-sIgE reactivity, the likelihood of positive tests for common ragweed, tree pollen, mugwort, peanut, soybean or Humulus scandens was less than $80 \%$. Patients with classes 4-6 CCD-sIgE reactivity, on the other hand, had an $85 \%$ to $100 \%$ chance of concomitant positivity for common ragweed, tree pollen, mugwort, peanut, soybean, and Humulus scandens (Figure 2B).

The optimal scale analysis showed that the distance between $\mathrm{CCD}$ and common ragweed or tree mix was the shortest, indicating a close relationship, whereas the distance between CCD and mugwort or soybean was the longest, indicating a weak relationship (Figure 3).

\section{Effects of the CCD Blocker on lgE-Binding to Plant Allergens}

After incubating $13 \mathrm{CCD}$-sIgE negative and $33 \mathrm{CCD}$-sIgE positive sera with the CCD inhibitor, all CCD-sIgE positive sera turned negative. Furthermore, as shown in Figures 4, $73.0 \%$ to $100 \%$ of the $\operatorname{sgE}$ tests for other plant allergens turned negative after inhibition, with the most common being Humulus scandens $(100 \%, 15 / 15)$, followed by mugwort and peanut $(85.2 \%, 23 / 27)$, ragweed $(81.5 \%, 22 / 27)$, soybean $(80.0 \%, 20 / 25)$, and tree pollen $(73.0 \%, 19 / 26)$. The scatter plot showing sIgE class transition after CCD inhibited is shown in Figure 5. No significant difference was found in the positivity rate for plant allergens in CCD-sIgE negative patients before and after inhibition (all $P>0.05$ ) 
A

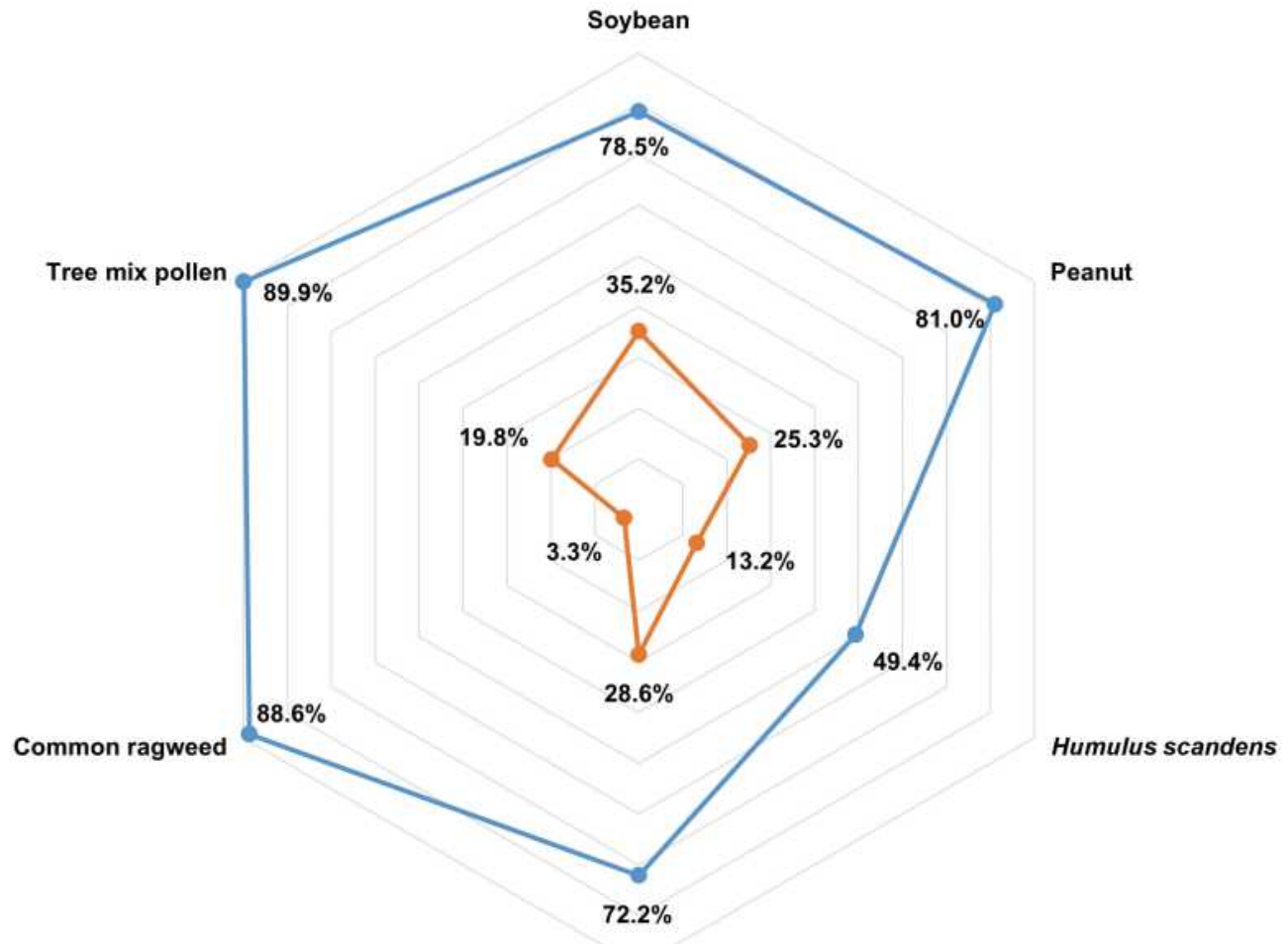

Mugwort

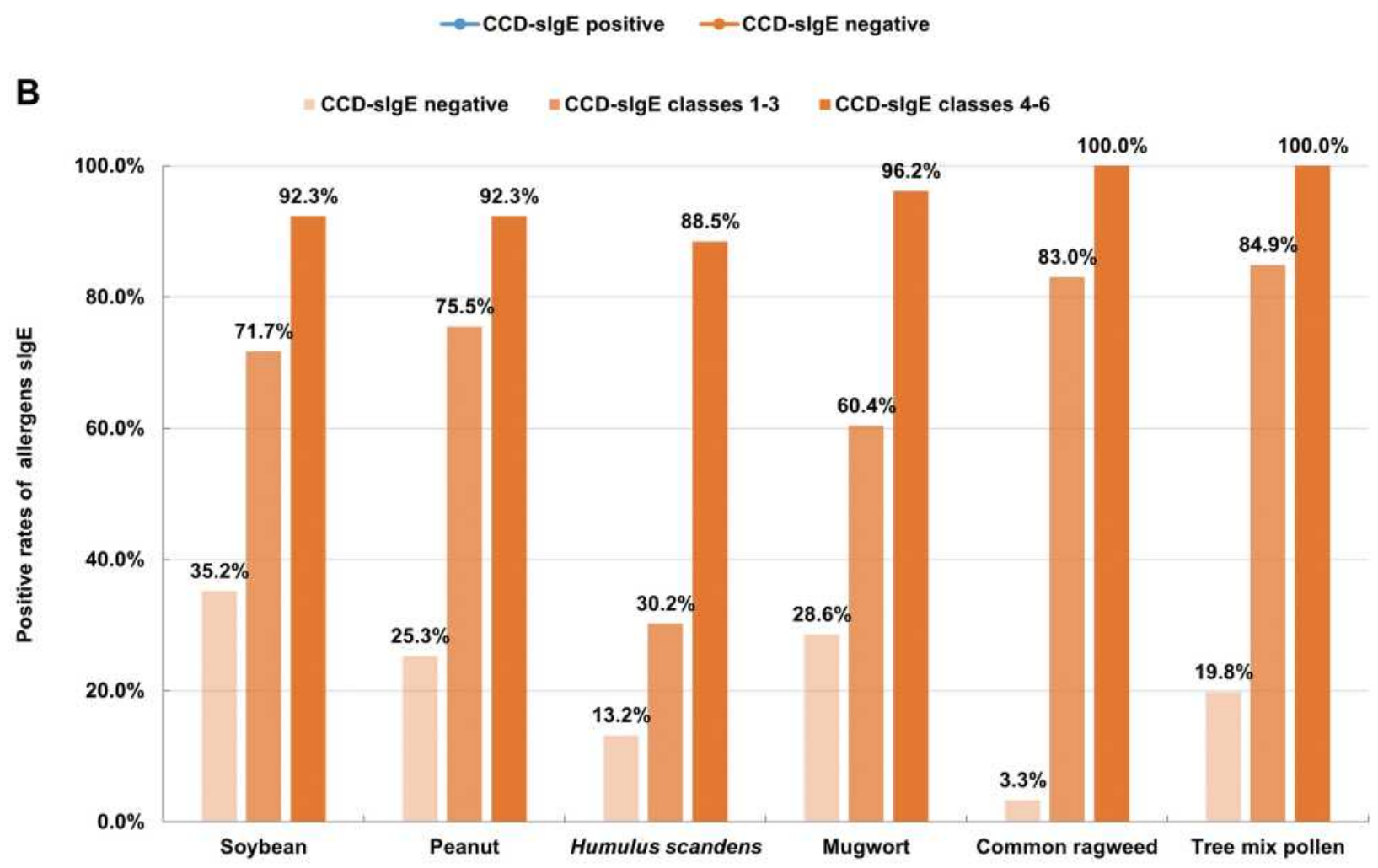

Figure 2 Relationship between plant allergen sensitisation and CCD-slgE. (A) Radar charts of plant allergens slgE in CCD slgE-negative or CCD slgE-positive patients, (B) the positivity of plant allergens slgE in patients with different CCD classes. CCD-slgE negative $(n=91), C C D$-slgE positive ( $n=79), C C D$-slgE classes I-3 ( $n=53), C C D$-slgE classes 4-6 $(n=26)$. 


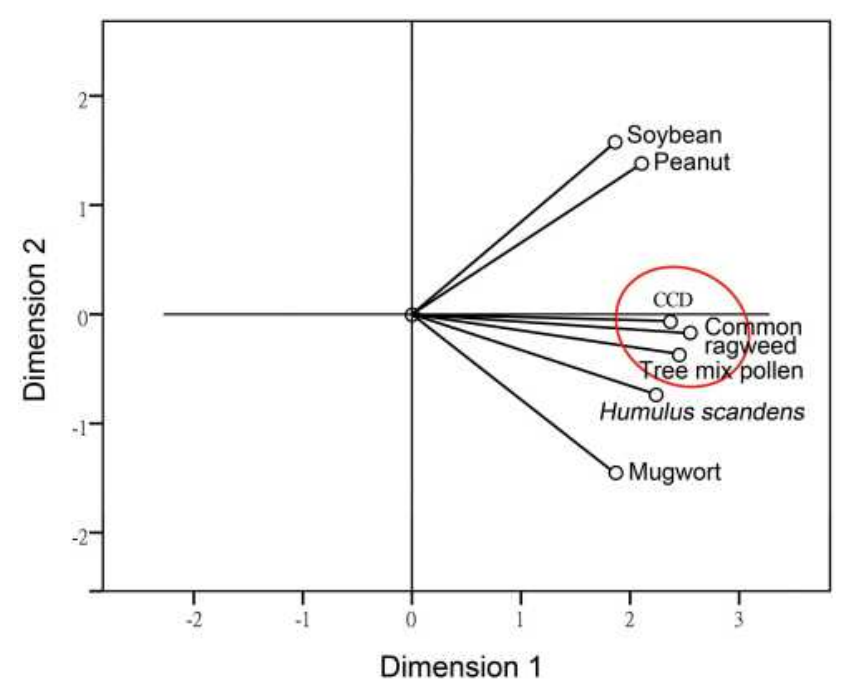

Figure 3 Optimal scale analysis of the slgE levels of CCD and plant allergens. The closer the distance between the points is, the greater the likelihood that they contain the same information, indicating a closer relationship between them. The close proximity of CCD and ragweed and tree pollen allergens indicated that they have the closest relationship (Cronbach's alpha $=95.6 \%$, suitable for this analysis).

\section{Discussion}

In this study from southern China, $66.3 \%$ of patients with SIgE positivity for pollen or plant allergens were sensitised to tree mix pollen, $61.4 \%$ to mugwort, $56.6 \%$ to ragweed, and $37.3 \%$ to Humulus scandens. Furthermore, sIgE levels of these allergens were concentrated in the classes $1-3$ in $65 \% \sim 96 \%$ of all patients. SIgE against CCDs was detected in 52.7\% of these patients. The proportions of adult patients sensitised to PAS (80.8\% vs $77.5 \%)$ and CCD (48.5\% vs $43.7 \%$ ) have no statistically significant differences with that of children. This means that CCD plays an important role in the sensitization of plant allergens in both children and adults. The positivity rate for plant food or pollen allergen sIgE was significantly higher in CCD-sIgE positive patients than in CCD-sIgE negative patients (Figure 3). This finding implies that, in CCDsIgE positive patients, the reactivity with the tested pollen and plant food allergens is primarily due to reactivity with $\mathrm{CCD}$ present on these allergens, rather than with the protein itself., ${ }^{3,16}$ The current findings also support CCD as a major cause of in vitro crossreactivity between pollen and food allergens, ${ }^{6}$ serving as a potential source of interference that causes nonspecific false positivity in in vitro tests. ${ }^{16,17}$ In this study, the positive rates of peanut and soybean were greater than $62 \%$, and approximately $70 \%$ of the patients were simultaneously positive for pollen allergen or CCD. Because recombinant allergen components do not have CCD, they may be able to eliminate the problem of cross-reactions caused by CCD. ${ }^{18}$ Although many allergen molecular tests can be used to diagnose individual pollen or plant food allergens, they have not been widely used in developing countries. CCD has long been known, ${ }^{4,19-21}$ and test CCD inhibition test has been performed for some time in basic research, ${ }^{22-24}$ but it has largely been ignored in clinical practice. Worm et al found that the prevalence of anti-CCD IgE in patients with food allergies ranged from $10 \%$ and $50 \%$ in the literature depending on the study and food examined (zucchini, celery, carrot, and tomato). ${ }^{25}$ Interestingly, IgE against CCD does not appear to cause any clinically relevant allergy symptoms in the majority of patients. ${ }^{4,26,27}$ In our study, all CCD SIgE was inhibited when the sera of patients were incubated with a CCD inhibitor. The pollen sIgE of Humulus scandens was also inhibited by $100 \%$, and serum $\operatorname{sigE}$ tests for peanut, soybean, mugwort and ragweed turned negative after using CCD inhibition in more than $80 \%$ of the patients. If the antigen of the truly sensitising component was present, it would not be completely suppressed by CCD inhibitor. $^{10,16}$ This finding implies that these pollen allergen sIgEs are likely false-positive reactions caused by CCD rather than sensitisation caused by real antigens, confirming that pollen allergens are not the most common allergens in southern China. Holzweber et al demonstrated that the CCD suppression test could improve allergen diagnosis accuracy. ${ }^{28}$ Based on our current findings, CCD inhibitors should be used for further testing after positive results for plant allergen sIgE are detected using the current allergen crude extract. However, the use of CCD inhibitors in all clinical tests requires consideration of cost and operational feasibility.

It is known whether each pollen $\operatorname{sigE}$ antibody binds directly to CCD-associated antigens. These findings, however, suggest that the reactivity to pollen allergens is entirely due to clinically irrelevant CCDSIgE antibodies. Attention should be given to the use of 

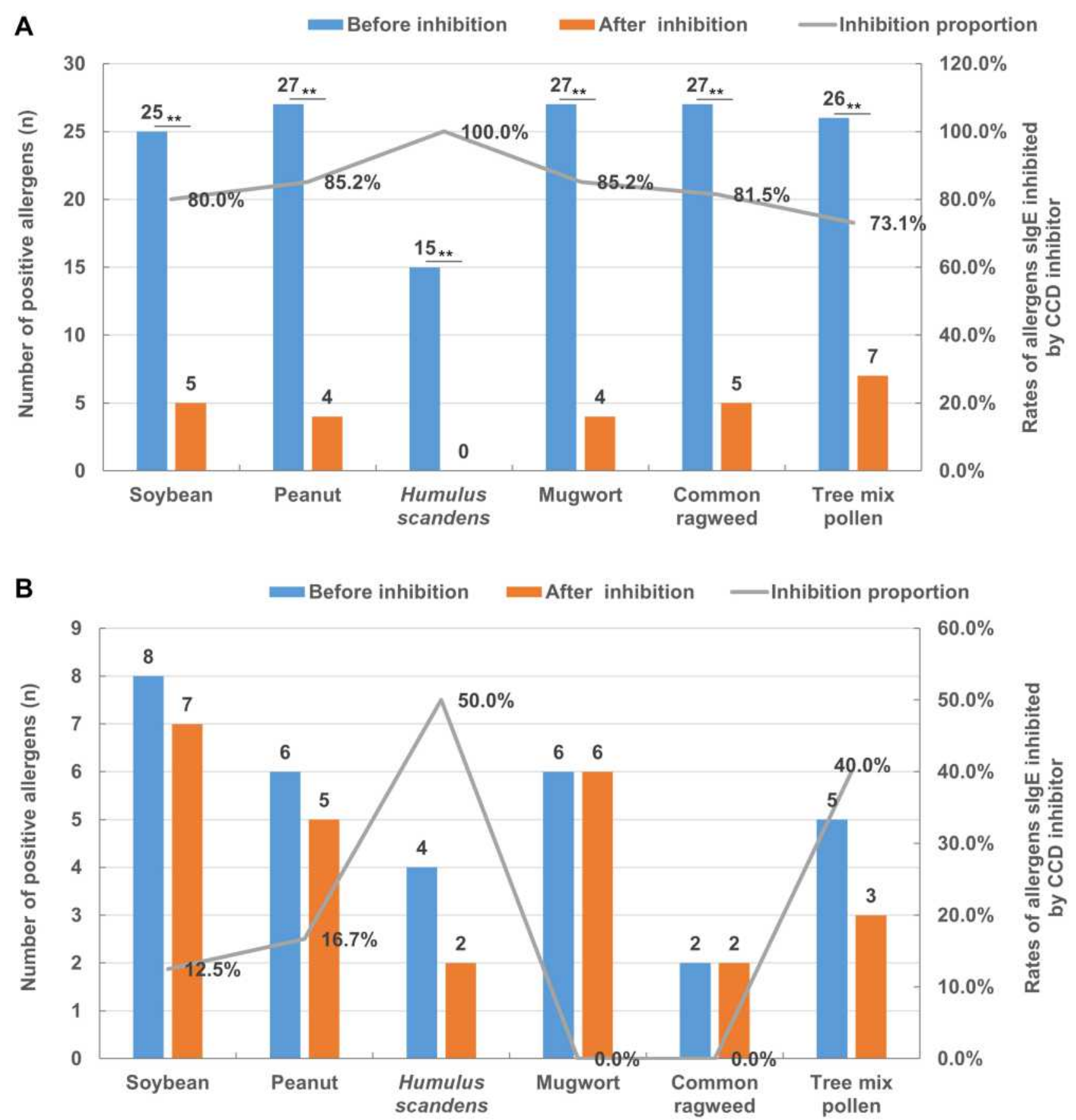

Figure 4 Changes in plant allergens before and after CCD inhibition. $(\mathbf{A})$ CCD-positive patients $(\mathrm{n}=33),(\mathbf{B}) \mathrm{CCD}$-negative patients $(\mathrm{n}=\mathrm{I7})$. $* * P<0.0 \mathrm{I}$, the positivity rate before and after inhibition was significantly different.

CCD inhibitors in diagnosing in vitro allergies because of their importance in diagnosing and treating local allergic diseases.

\section{Declaration}

The abstract of this work was presented at 2019 ERS International Congress. 

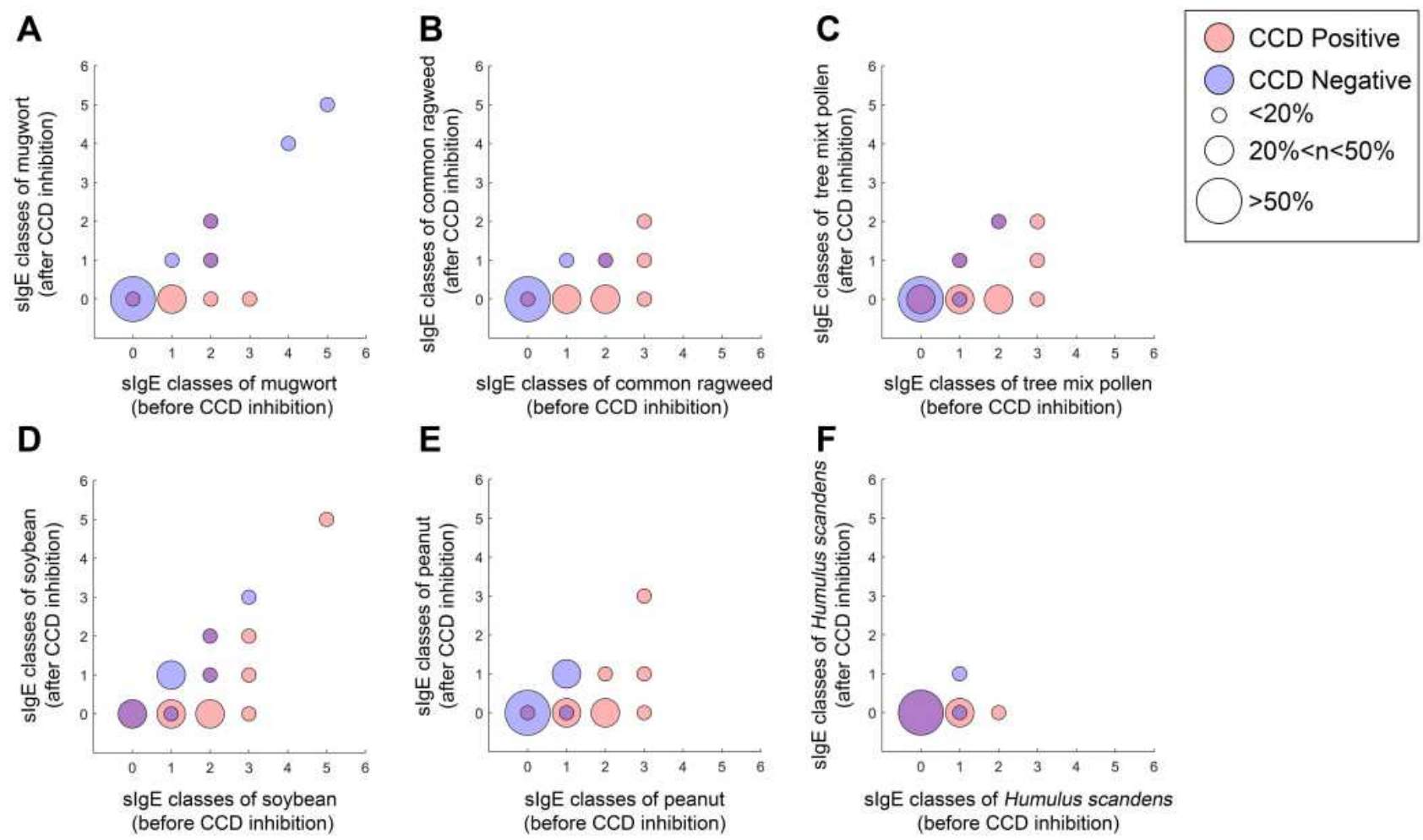

Figure 5 The scatter diagram of plant allergen slgE classes before or after CCD inhibited. (A) for mugwort (CCD positive/negative $n=27 / 6)$, $(\mathbf{B})$ for common ragweed (CCD positive/negative $n=27 / 2$ ), (C) for tree mix pollen (CCD positive/negative $n=26 / 5)$, (D) for soybean (CCD positive/negative $n=25 / 8)$, (E) for peanut (CCD positive/ negative $n=27 / 6$ ), (F) for Humulus scandens (CCD positive/negative $n=15 / 4$ ).

\section{Acknowledgments}

This study was supported by the National Natural Science Foundation of China (81802076 and 81871736), the National Key Technology R\&D Program (2018YFC1311900), the Guangdong Science and Technology Foundation (2019B030316028), SKLRD-MS-201906, and the Guangzhou Science and Technology Foundation (201804020043). Thanks the Biobank for Respiratory Diseases in the National Clinical Research Center for Respiratory Disease (BRD-NCRCRD, Guangzhou, Southern China).

\section{Author Contributions}

All authors made a significant contribution to the work reported, whether that is in the conception, study design, execution, acquisition of data, analysis and interpretation, or in all these areas; took part in drafting, revising or critically reviewing the article; gave final approval of the version to be published; have agreed on the journal to which the article has been submitted; and agree to be accountable for all aspects of the work. Cocorresponding authors: Jinping Zheng and Baoqing Sun.

\section{Funding}

This study was supported by the National Natural Science Foundation of China (81802076 and 81871736), the National Key Technology R\&D Program (2018YFC1311900), the Guangdong Science and Technology Foundation (2019B030316028), SKLRD-MS -201906, and the Guangzhou Science and Technology Foundation (201804020043).

\section{Disclosure}

Jinping Zheng reports grants from the Department of Science and Technology during the conduct of the study. The authors reported no other potential conflicts of interest for this work.

\section{References}

1. Costongs GM, Bas BM. The first fully automated allergy analyser UniCAP: comparison with IMMULITE for allergy panel testing. Eur J Clin Chem Clin Biochem. 1997;35(11):885-888.

2. Hidenori Y, Hiroshi Y, Yuma M, et al. Involvement of cross-reactive carbohydrate determinants-specific IgE in pollen allergy testing. Asia Pac Allergy. 2017;7:29-36. doi:10.5415/apallergy.2017.7.1.29

3. Aalberse RC, Akkerdaas J, van Ree R. Cross-reactivity of $\operatorname{IgE}$ antibodies to allergens. Allergy. 2001;56(6):478-490. doi:10.1034/j.13989995.2001.056006478.x 
4. Foetisch K, Westphal S, Lauer I, et al. Biological activity of IgE specific for cross-reactive carbohydrate determinants. J Allergy Clin Immunol. 2003;111(4):889-896. doi:10.1067/mai.2003.173

5. Sturm GJ, Jin C, Kranzelbinder B, et al. Inconsistent results of diagnostic tools hamper the differentiation between bee and vespid venom allergy. PLoS One. 2011;6(6):e20842. doi:10.1371/journal. pone. 0020842

6. Malandain H, Giroux F, Cano Y. The influence of carbohydrate structures present in common allergen sources on specific IgE results. Eur Ann Allergy Clin Immunol. 2007;39:216-220.

7. Jin C, Nitsch S, Hemmer W, Altmann F. Improving allergy diagnosis by removal of CCD-specific IgE from patients' sera. Allergy. 2009;64:30.

8. Renz H, Biedermann T, Bufe A, et al. AWMF-leitlinie in vitro allergie diagnostik. Allergo J. 2010;19:110-128. doi:10.1007/BF03362255

9. Jappe U, Raulf-Heimsoth M. The significance of cross-reactive carbohydrate determinants (CCD) for allergy diagnosis. Allergologie. 2008;31:82-90. doi:10.5414/ALP31082

10. Jappe U, Raulf-Heimsoth M, Hoffmann M, et al. In vitro hymenoptera venom allergy diagnosis: improved by screening for cross-reactive carbohydrate determinants and reciprocal inhibition. Allergy. 2006;61 (10):1220-1229. doi:10.1111/j.1398-9995.2006.01232.x

11. Luo W, Huang $H$, Zheng $P$, et al. Major grass pollen allergens and components detected in a southern Chinese cohort of patients with allergic rhinitis and/or asthma. Mol Immunol. 2016;78:105-112. doi:10.1016/j.molimm.2016.08.013

12. Brożek JL, Bousquet J, Agache I, et al. Allergic Rhinitis and its Impact on Asthma (ARIA) guidelines-2016 revision. J Allergy Clin Immunol. 2017;140(4):950-958. doi:10.1016/j.jaci.2017.03.050

13. Global Initiative for Asthma. Global strategy for asthma management and prevention; 2015. Available from: www.ginasthma.org. Accessed April 13, 2021.

14. Zeng G, Luo W, Zehong W, et al. A cross-sectional observational study on allergen-specific IgE positivity in a southeast coastal versus a southwest inland region of china. Sci Rep. 2017;30:9593. doi:10.1038/s41598-017-10109-3

15. Haisheng H, Huang Z, Luo W, et al. Allergen diagnosis strategy: an experimental application of different methods in Guangzhou, Southern China. Sci Prog. 2021;104:1-14.

16. Altmann F. The role of protein glycosylation in allergy. Int Arch Allergy Immunol. 2007;142(2):99-115. doi:10.1159/000096114

17. Zafred D, Nandy A, Pump L, Kahlert H, Keller W. Crystal structure and immunologic characterization of the major grass pollen allergen Phl p 4. J Allergy Clin Immunol. 2013;132(3):696-703. doi:10.1016/ j.jaci.2013.03.021

18. Seismann H, Blank S, Braren I, et al. Dissecting cross-reactivity in hymenoptera venom allergy by circumvention of alpha-1,3-core fucosylation. Mol Immunol. 2010;47:799-808. doi:10.1016/j. molimm.2009.10.005
19. Cecioni S, Faure S, Darbost U, et al. Selectivity among two lectins: probing the effect of topology, multivalency and flexibility of "clicked" multivalent glycoclusters. Chemistry. 2011;17 (7):2146-2159. doi:10.1002/chem.201002635

20. Van der Veen MJ, van Ree R, Aalberse RC, et al. Poor biologic activity of cross-reactive IgE directed to carbohydrate determinants of glycoproteins. J Allergy Clin Immunol. 1997;100(3):327-334. doi:10.1016/S0091-6749(97)70245-8

21. Tretter V, Altmann F, Kubelka V, Marz L, Becker WM. Fucose alpha 1,3-linked to the core region of glycoprotein $\mathrm{N}$-glycans creates an important epitope for $\mathrm{IgE}$ from honeybee venom allergic individuals. Int Arch Allergy Immunol. 1993;102:259-266. doi:10.1159/ 000236534

22. Bencurova M, Hemmer W, Focke-Tejkl M, Wilson IB, Altmann F. Specificity of $\mathrm{IgG}$ and $\mathrm{IgE}$ antibodies against plant and insect glycoprotein glycans determined with artificial glycoforms of human transferrin. Glycobiology. 2004;14:457-466. doi:10.1093/glycob/ cwh058

23. Wilson IB, Harthill JE, Mullin NP, Ashford DA, Altmann F. Core alpha1,3-fucose is a key part of the epitope recognized by antibodies reacting against plant $\mathrm{N}$-linked oligosaccharides and is present in a wide variety of plant extracts. Glycobiology. 1998;8:651-661. doi:10.1093/glycob/8.7.651

24. Jin C, Focke M, Leonard R, Jarisch R, Altmann F, Hemmer W. Reassessing the role of hyaluronidase in yellow jacket venom allergy. J Allergy Clin Immunol. 2010;125:184-190. doi:10.1016/j. jaci.2009.08.037

25. Worm M, Jappe U, Kleine-Tebbe J, et al. Food allergies resulting from immunological cross-reactivity with inhalant allergens. Allergo $J$ Int. 2014;23(1):1-16. doi:10.1007/s40629-014-0004-6

26. Malandain H. IgE-reactive carbohydrate epitopes-classification, cross-reactivity, and clinical impact. Eur Ann Allergy Clin Immunol. 2005;37:122-128

27. Mari A, Ooievaar-de Heer P, Scala E, et al. Evaluation by double-blind placebo-controlled oral challenge of the clinical relevance of IgE antibodies against plant glycans: oral challenge with transgenic glycosylated proteins. Allergy. 2008;63(7):891-896. doi:10.1111/j.1398-9995.2008.01703.x

28. Holzweber F, Svehla E, Fellner W, et al. Inhibition of IgE binding to cross-reactive carbohydrate determinants enhances diagnostic selectivity. Allergy. 2013;68:1269-1277. doi:10.1111/all.12229

\section{Publish your work in this journal}

The Journal of Asthma and Allergy is an international, peer-reviewed open-access journal publishing original research, reports, editorials and commentaries on the following topics: Asthma; Pulmonary physiology; Asthma related clinical health; Clinical immunology and the immunological basis of disease; Pharmacological interventions and new therapies. The manuscript management system is completely online and includes a very quick and fair peer-review system, which is all easy to use. Visit http://www.dovepress.com/testimonials.php to read real quotes from published authors. 